\title{
Impairment of Endothelium-dependent Dilation Is an Early Event in Children with Familial Hypercholesterolemia and Is Related to the Lipoprotein (a) Level
}

\author{
Keld E. Sorensen, * David S. Celermajer, * Dimitri Georgakopoulos, * Geoffrey Hatcher, ${ }^{*}$ \\ D. John Betteridge, ${ }^{3}$ and John E. Deanfield* \\ ${ }^{*}$ Cardiothoracic Unit, Hospital for Sick Children, London WC1N 3JH; ${ }^{\ddagger}$ Royal Alexandra Hospital \\ for Sick Children, Brighton BNI 3JN; and ${ }^{\S}$ Middlesex Hospital, London WIN 8AA, United Kingdom
}

\section{Abstract}

Familial hypercholesterolemia is associated with premature atherosclerosis. Since endothelial dysfunction is an early event in atherogenesis, we used a noninvasive method to assess endothelial function in the systemic arteries of $\mathbf{3 0}$ children aged 7-17 yr (median 11 ) with familial hypercholesterolemia ( 2 homozygotes, 28 heterozygotes, total cholesterol $240-696 \mathrm{mg} /$ dl) and 30 healthy age- and sex-matched controls. Using high resolution ultrasound, the diameter of the superficial femoral artery was measured at rest, in response to reactive hyperemia (with increased flow causing endothelium-dependent dilation), and after sublingual glyceryltrinitrate (causing endothelium-independent vasodilation).

Flow-mediated dilation was present in the controls $\mathbf{( 7 . 5} \pm \mathbf{0 . 7} \%$ ) but was impaired or absent in the hypercholesterolemic children ( $1.2 \pm 0.4 \%, P<0.0001)$. Total cholesterol was inversely correlated with flow-mediated dilation $(r=-0.61$, $P<0.0001$ ). In the hypercholesterolemic children, flow-mediated dilation was inversely related to the lipoprotein(a) level $(r=-0.61, P=0.027$ ) but not to other lipid fractions. Glyceryltrinitrate-induced dilation was present in all subjects but was lower in the hypercholesterolemia group $(10.0 \pm 0.6 \%$ vs $12.4 \pm 0.8 \%, P=0.023$ ).

Thus, impaired endothelium-dependent dilation is present in children with familial hypercholesterolemia as young as $7 \mathbf{~ y r}$ of age and the degree of impairment is related to the lipoprotein(a) level. (J. Clin. Invest. 1994. 93:50-55.) Key words: atherosclerosis • atherogenesis • ultrasound $\bullet$ cholesterol $\bullet$ flow

\section{Introduction}

Although the clinical complications of atherosclerosis occur in adult life, the process of atherogenesis begins in childhood (1, 2 ). Hypercholesterolemia is an important risk factor for the early structural changes of atherosclerosis ( 3 ) and for cardiovascular morbidity and mortality in later life (4). In autopsy stud-

This work was presented in abstract form at the Annual Scientific Sessions of the American College of Cardiology in Anaheim, CA, 14-18 March 1993.

Address correspondence to Dr. John E. Deanfield, Cardiothoracic Unit, Hospital for Sick Children, Great Ormond Street, London WC1N 3JH, United Kingdom.

Received for publication 18 June 1993 and in revised form $19 \mathrm{Au}$ gust 1993.

J. Clin. Invest.

(C) The American Society for Clinical Investigation, Inc.

0021-9738/94/01/0050/06\$2.00

Volume 93, January 1994, 50-55 ies of young adults, a significant positive correlation between LDL cholesterol and the extent of atherosclerosis in the aorta and coronary circulation has been documented (3). Furthermore, a recent long-term prospective study has shown a strong association between the cholesterol level in young adult life and the later risk of cardiovascular disease (5). In particular, if levels of LDL cholesterol and lipoprotein (a) $[\mathrm{Lp}(\mathrm{a})]^{1}$ are elevated, the risk of coronary disease is markedly increased (6).

Endothelial dysfunction is an important early event in atherogenesis $(7,8)$. Both spatial and temporal correlations between endothelial dysfunction and coronary atherosclerosis have been shown in animal models and in humans (9-11). Endothelial injury has been induced experimentally by hypercholesterolemia, particularly the LDL component (12). In clinical studies using invasive techniques, hypercholesterolemic adults have been shown to have impaired endothelium-dependent responses to a variety of pharmacologic stimuli, including infusions of acetylcholine $(13,14)$.

We have tested whether endothelial dysfunction can be demonstrated at a much earlier stage of the disease process, in the systemic arteries of young children with familial hypercholesterolemia. In addition, we have examined the relationship between disturbed arterial physiology and lipid subfractions at a stage when intervention might have the greatest benefit.

\section{Methods}

Subjects. 30 children ( 17 girls, 13 boys) aged $7-17$ yr (median 11 ) with familial hypercholesterolemia ( FH) were studied. All were asymptomatic, normotensive, nondiabetic, and nonsmokers. $\mathrm{FH}$ was defined as total plasma cholesterol $>268 \mathrm{mg} / \mathrm{dl}$ or LDL cholesterol $>196 \mathrm{mg} / \mathrm{dl}$ with tendon xanthomas in the patient or in a relative (15). 28 of these children were heterozygotes and 2 were homozygotes. All 30 were prescribed lipid-lowering diet. Drug therapy was prescribed at the discretion of each patient's physician, and 12 were receiving lipid-lowering drugs ( 7 cholestyramine, 5 3-hydroxy-3-methylglutaryl CoA reductase inhibitors) for $1-7 \mathrm{yr} .30$ healthy children ( 17 girls, 13 boys, aged 5-15, median $11 \mathrm{yr}$ ) served as controls. All were normotensive, nonsmokers, and had no family history of premature vascular disease. Each subject and/or their parents gave informed consent to the study, which was approved by the local committee on ethical practice.

Lipid analysis. Total plasma cholesterol was measured in all 60 subjects (FH group and controls) using the cholesterol C-system high performance cholesterol oxidase 4-aminophenazone method (Boehringer Mannheim Diagnostica GmbH, Mannheim, Germany). Measurement of plasma lipids and lipoproteins in the 28 children with heterozygous FH was performed on EDTA plasma after a 14-h overnight fast. Plasma triglyceride was measured using the glyceryl phosphate oxidase 4-aminophenazone high performance enzymatic colori-

1. Abbreviations used in this paper: $\mathrm{FH}$, familial hypercholesterolemia; FMD, flow-mediated dilation; GTN, glyceryltrinitrate; $\operatorname{Lp}(\mathrm{a})$, lipoprotein (a); SFA, superficial femoral artery. 
metric test (Boehringer Mannheim Diagnostica GmbH). HDL chólesterol was measured after precipitation of apoprotein B containing lipoproteins and LDL cholesterol was calculated according to the Friedwald formula (16). Lp(a) was measured using an ELISA method (Immuno GmbH, Sevenoaks, United Kingdom).

Study design. Arterial, endothelial, and smooth muscle function was studied noninvasively, as described previously (17). High resolution ultrasound was used to measure changes in arterial diameter in response to reactive hyperemia (with increased flow producing an endothelium-dependent stimulus to vasodilation ) and to glyceryltrinitrate (GTN, an endothelium-independent vasodilator) (128XP/10 with a $7.0 \mathrm{MHz}$ linear array transducer; Acuson, Mountain View, California).

The subject rested in the supine position for $10 \mathrm{~min}$ before the first scan and remained supine throughout the study. The superficial femoral artery (SFA) was scanned in longitudinal section and the center of the vessel was identified when the clearest images of the anterior and posterior walls of the artery were obtained. The transmit zone was set to the level of the anterior vessel wall. Depth and gain settings were optimized to identify the lumen to vessel wall interface. Images were magnified with the resolution box function leading to a television line width of $\sim 0.065 \mathrm{~mm}$. Machine settings were kept constant during each study.

Flow increase was induced by inflation of a blood pressure tourniquet placed around the thigh, distal to the target artery, to $\sim 250$ $\mathrm{mmHg}$. The cuff was released after 4-5 min and the artery was scanned continuously for $30 \mathrm{~s}$ before and $90 \mathrm{~s}$ after cuff deflation. $10 \mathrm{~min}$ later, a second resting scan was recorded. GTN ( $400 \mu \mathrm{g}$ spray) was then administered sublingually and the artery was scanned 3-4 min later.

Phantom studies performed in our laboratory have confirmed that changes in diameters of $0.1-0.2 \mathrm{~mm}$ can be detected accurately with this method (18). Furthermore, we (17) and others $(19,20)$ have found a low coefficient of variation for measurements of arterial diameter (interobserver error) and a high correlation between consecutive control measurements within a study.

Data analysis. Scans were recorded on super-VHS tapes and arterial diameters were measured directly from the tape by two independent observers "blinded" to the scan sequence and the identity of the subject. Vessel diameters were measured from the anterior to the posterior interface between media and adventitia (the " $m$-line") at a fixed distance from an anatomical marker, usually the flow divider between the superficial and profunda branches of the common femoral artery. The mean diameter was calculated from four cardiac cycles incident with the R-wave on the electrocardiogram (ECG). For the hyperemia scan, vessel diameter was measured $45 \mathrm{~s}$ after cuff release (Fig. 1). Diameter changes were derived as percent change relative to the first baseline scan (100\%). Baseline blood flow (measured during the first baseline scan) was estimated by multiplying angle-corrected, pulsed Doppler recordings of the flow-velocity integral by $\pi$ and the square of the radius of the artery. Reactive hyperemia was calculated as the maximum flow measured during the first $15 \mathrm{~s}$ after cuff deflation divided by the baseline flow.

Statistics. Descriptive data are expressed as mean \pm SEM. The FH and control groups were compared using two sample $t$ tests. For the whole group, the relationships between the dependent variables flowmediated dilation (FMD) and GTN-induced dilation and sex, vessel size, and total cholesterol level were explored by both univariate and multivariate regression analysis. For the 28 children with hetrozygous FH, the relationship between FMD and GTN-induced dilation and age, sex, vessel size, cholesterol, triglycerides, HDL, LDL, apolipoproteins $A$ and $B$, and $L p(a)$ levels was explored by univariate analysis and multiple regression models. The distribution of $\operatorname{Lp}(\mathrm{a})$ levels was nor-
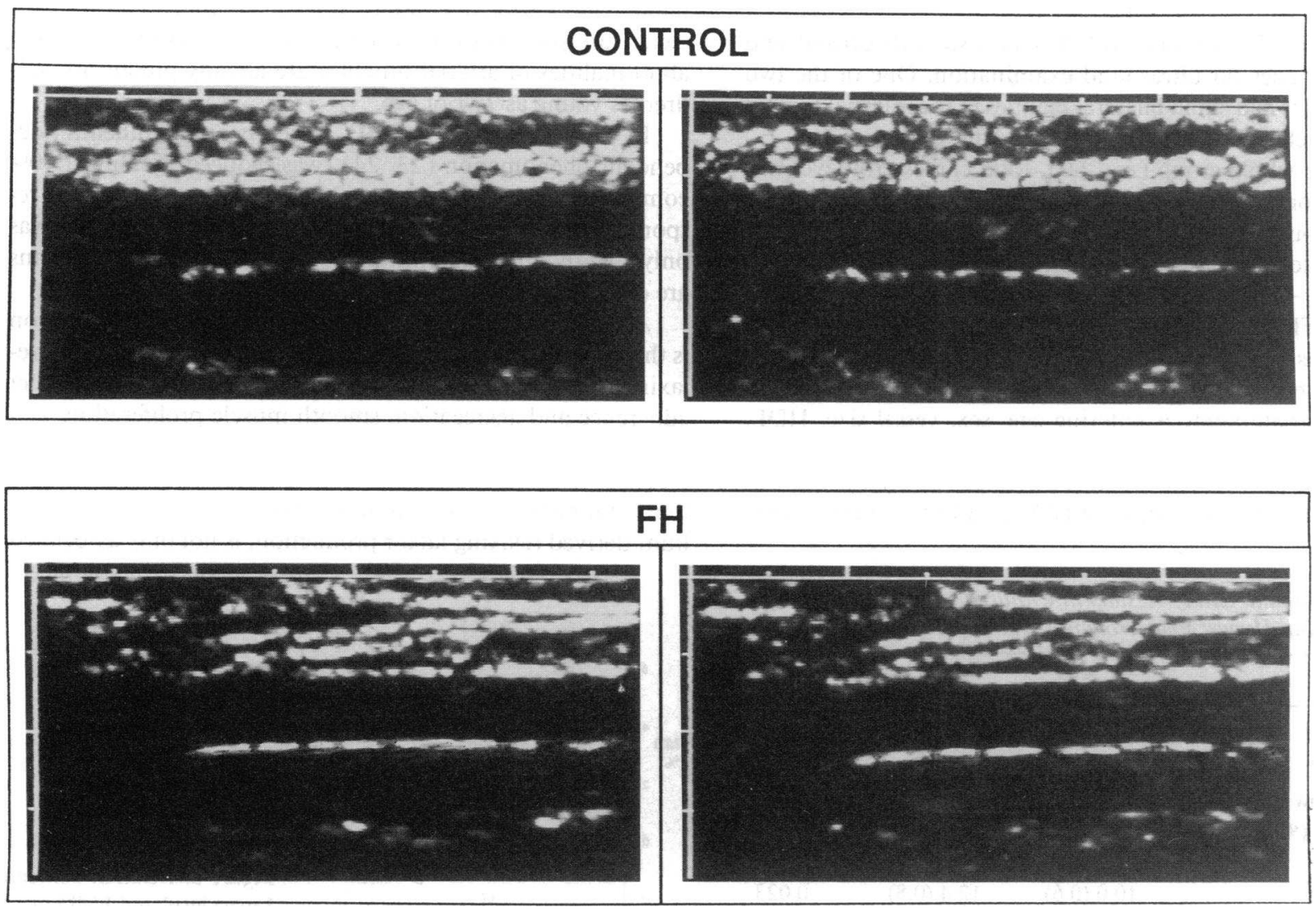

Figure 1. Scans from the superficial femoral artery of a control child (top panels) and from a child with FH (lower panels), showing the artery at rest (left) and after reactive hyperemia (right). In the control subject, the artery dilates $\sim 10 \%$, and in the child with FH there is no increase in arterial diameter during increased flow. 
mal in this group except for three subjects who had very high levels $(>50 \mathrm{mg} / \mathrm{dl}$ ); therefore, the analyses were repeated excluding these three children. Statistical significance was inferred at a $P$ value of $<0.05$.

\section{Results}

Lipid data. Total plasma cholesterol in the control subjects was $164 \pm 27 \mathrm{mg} / \mathrm{dl}$ ( range 107-204). Total cholesterol was significantly increased in the FH group $(312 \pm 16 \mathrm{mg} / \mathrm{dl}, P<0.0001)$. In the FH children, HDL level was $47 \pm 1.4 \mathrm{mg} / \mathrm{dl}$ (range $31-$ 61 ) and LDL level was $224 \pm 11 \mathrm{mg} / \mathrm{dl}$ (range 151-374). Triglyceride levels were normal $(86 \pm 6 \mathrm{mg} / \mathrm{dl})$. Apolipoprotein A was $130 \pm 4 \mathrm{mg} / \mathrm{dl}$ (range 96-195). Apolipoprotein B was $132 \pm 6 \mathrm{mg} / \mathrm{dl}$ ( range $70-215$ ). $\mathrm{Lp}(\mathrm{a})$ was $24.8 \pm 3.2 \mathrm{mg} / \mathrm{dl}$ ( $\mathrm{me}-$ dian 18.5, interquartile range 13-31, range 7.2-70).

Vascular studies. Vessel size was similar in both groups ( $4.83 \mathrm{~mm}$ in control vs $4.79 \mathrm{~mm}$ in FH subjects, $P=0.807$ ). The mean resting blood flow in the SFA was $>269 \pm 24 \mathrm{ml} /$ min in controls and $232 \pm 17 \mathrm{ml} / \mathrm{min}$ in the $\mathrm{FH}$ group $(P=$ $0.138)$. The mean flow increase observed immediately after cuff deflation was $414 \pm 19 \%$ in the control and $419 \pm 20 \%$ in the FH children $(P=0.876)$ ( Table I).

In the arteries of the control children, FMD was $7.5 \pm 0.7 \%$ and GTN-induced dilation was $12.4 \pm 0.8 \%$ (Fig. 2). FMD was absent $(<2 \%)$ in 3 of these 30 children. In the FH subjects,

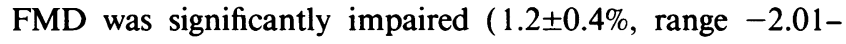
$5.8 \%, P<0.0001$, Table I). In 22 subjects (73\%) FMD was absent. GTN-induced dilation was mildly attenuated in $\mathrm{FH}$ subjects compared with controls $(10.0 \pm 0.6 \%$, range $4.0-$ $16.5 \%, P=0.023$ ) (Table I, Fig. 2).

29 of the 30 children with FH had smooth carotid and femoral arteries on ultrasound examination. One of the two homozygotes had small bilateral plaques in the internal carotid artery, which did not cause flow disturbance.

For the whole group $(n=60)$, total cholesterol level was inversely correlated with FMD $(r=-0.58, P<0.0001)$ but not with GTN-induced dilation. A similar result was obtained on multivariate analysis (total cholesterol related inversely to FMD, $r=-0.61, P<0.0001$ ). For the 28 heterozygous children with FH, the only significant correlate of FMD on univariate analysis was $\operatorname{Lp}(\mathrm{a})(r=-0.51, P<0.01)$ (Fig. 3). This inverse correlation between FMD and $\operatorname{Lp}(\mathrm{a})$ was still observed on multivariate analysis entering age, sex, vessel size, HDL, LDL, triglycerides, and $\operatorname{Lp}(\mathrm{a})(r=-0.61, P=0.027)$ ( Table II). Similar results were obtained if total cholesterol was entered into this model instead of HDL and LDL. Furthermore,

Table I. Vascular Study Results in Children with Familial Hypercholesterolemia and Controls

\begin{tabular}{lccc}
\hline & FH & Controls & $P$ \\
\hline Age $(y r)$ & $10.9(0.46)$ & $10.9(0.45)$ & 0.92 \\
Vessel size* & $4.79(0.10)$ & $4.83(0.10)$ & 0.807 \\
Baseline flow $(\mathrm{ml} / \mathrm{min})^{*}$ & $232(17)$ & $269(24)$ & 0.138 \\
Hyperemia (\%) & $419(20)$ & $414(19)$ & 0.876 \\
FMD (\%) & $1.2(0.4)$ & $7.5(0.7)$ & $<0.0001$ \\
GTN (\%) & $10.0(0.6)$ & $12.4(0.8)$ & 0.023 \\
\hline
\end{tabular}

Results are expressed as mean $\pm \mathrm{SE}$. * As measured during the first (resting) scan.
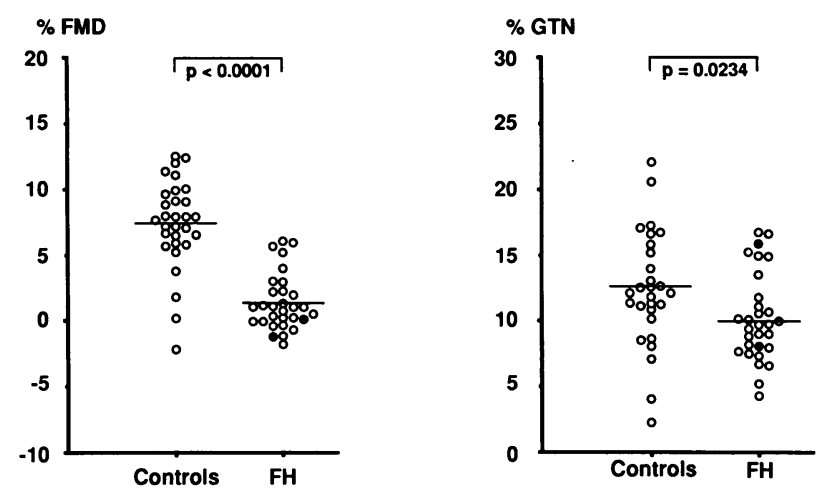

Figure 2. Flow-mediated and GTN-induced dilation in the controls and children with FH. Horizontal lines, group means; $\bullet$ subjects with homozygous $\mathrm{FH}$.

when these multivariate analyses were repeated excluding the three children with very high $\operatorname{Lp}(\mathrm{a})$ levels, almost identical results were obtained ( $\mathrm{Lp}[\mathrm{a}]$ inversely related to FMD, $r=-0.55, P=0.029$ ).

\section{Discussion}

Familial hypercholesterolemia is an autosomal, dominant lipoprotein disorder with a prevalence of $\sim 1$ :500 in its heterozygous form (21). Heterozygotes have a 100 -fold increased risk of premature death from coronary heart disease (15). In homozygous $\mathrm{FH}$, clinical coronary disease can occur in childhood. The metabolic consequences of the LDL-receptor deficiency are present from birth (22) and our results show that abnormalities of arterial function are already present in children as young as $7 \mathrm{yr}$ of age.

In children with FH, FMD, which is an endothelium-dependent phenomenon $(23,24)$, was reduced by almost $90 \%$ compared with healthy controls. In contrast, the GTN response, which tests smooth muscle relaxation directly, was only slightly attenuated in the FH group. These observations are consistent with endothelial dysfunction.

A major functional consequence of endothelial dysfunction is the inability to produce and release endothelium-derived relaxing factor, a local vasodilator, which also inhibits platelet adherence and aggregation, smooth muscle proliferation, and endothelial cell-leukocyte interactions, all of which are important early events in atherogenesis (8). Recent evidence suggests that administration of L-arginine, the substrate for endothelium-derived relaxing factor production, is not only associated

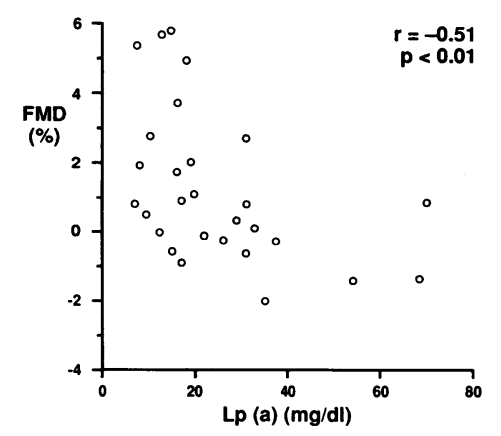

Figure 3. Relation between Lp(a) level and FMD in the superficial femoral arteries of the children with heterozygous FH $(n=28)$. 
Table II. Multivariate Regression Analysis for Determinants of Flow-mediated Dilation in 28 Children with Heterozygous Familial Hypercholesterolemia

\begin{tabular}{lcc}
\hline \multicolumn{1}{c}{ Variable } & Partial regression coefficient & $P$ \\
\hline Age & +0.41 & 0.19 \\
Sex & -0.25 & 0.34 \\
Vessel size & -0.37 & 0.18 \\
LDL & -0.11 & 0.60 \\
HDL & -0.08 & 0.68 \\
Triglycerides & -0.08 & 0.74 \\
Lp(a) & -0.61 & 0.027 \\
\end{tabular}

with preserved endothelium-dependent responses but is also antiatherogenic (25). This implies that endothelial dysfunction is not merely a marker of early atherosclerosis but may be intimately involved in its pathogenesis.

Hypercholesterolemia, especially LDL cholesterol, is known to cause endothelial injury in vitro, with attenuation of the normal endothelium-dependent relaxation occurring long before the formation of atherosclerotic lesions (13, 26-29). In vitro, native LDL cholesterol appears to impair reversibly endothelium-dependent dilation minutes after exposure (30), and oxidized LDL cholesterol causes slowly developing and apparently irreversible inhibition of endothelium-dependent relaxation $(31,32)$. In experimental animals with diet-induced hypercholesterolemia, arteries show impaired vasodilation to acetylcholine in the absence of atheromatous lesions $(30,33)$. In clinical studies, selective loss of endothelium-dependent vasodilation to acetylcholine has been documented in hypercholesterolemic adults with angiographically normal coronary arteries (34) and has been found to be associated with the degree of elevation of serum cholesterol (35). Our findings show that the damaging interaction between hypercholesterolemia and the endothelium may begin even earlier, in the first decade of life.

We have investigated which lipid subfractions might be related to early vascular injury in these children. In our relatively small group, HDL and LDL levels were not correlated with impaired endothelial function. However, a significant relation between LP(a) and FMD was observed, independent of the total cholesterol level. $\operatorname{Lp}(\mathrm{a})$ has recently been documented as a risk factor for premature atherosclerosis (36-38). Patients with FH suffering from coronary heart disease have higher $\mathrm{Lp}$ (a) levels than $\mathrm{FH}$ patients free of coronary artery disease $(39,40)$. The mechanism by which high levels of $\operatorname{Lp}(a)$ are related to atherosclerosis is unclear, but hypotheses include inhibition of plasminogen activity (41) and alteration of LDLmediated delivery of cholesterol to the vessel wall (6). Our data represent the first evidence that elevated $L p(a)$ levels may be related to endothelial injury in vivo.

The simple noninvasive test used in this study enables accurate and reproducible assessment of vascular responses in humans (17). Flow-mediated dilation of large systemic arteries depends on the ability of the healthy endothelium to release relaxing factors $(23,42)$. We have induced a condition of increased flow by distal circulatory arrest followed by reperfusion. This method has been shown to produce dilation of the proximal artery by increase in flow rather than by altering cardiovascular reflexes, changing arterial distending pressure, or via the release of ischemic metabolites (43). In our laboratory, we have demonstrated that equivalent cuff occlusion of the contralateral limb has no effect on ipsilateral arterial diameter. In contrast, GTN produces vasodilation by acting directly on smooth muscle. GTN was an effective vasodilator in both controls and FH children, although there was a modest impairment of GTN response in the latter group. A similar trend has been observed previously in small groups of hypercholesterolemic adults with either angiographically normal coronary arteries (34) or minimal coronary irregularities (44) and also in the superficial femoral artery of patients with mild atherosclerosis (45). The vasodilator response observed after GTN administration is a function of both direct smooth muscle relaxation and the response to the hyperemia caused by GTN-induced dilation of the resistance vessels (46). Loss of this flow component to the GTN response may explain the mildly impaired response seen in the FH group. In the setting of hypercholesterolemia, superoxide anions have been shown to inactivate nitric oxide and this also might contribute to the reduced responses of our FH patients, both to increased flow and to an exogenously administered nitrovasodilator (47). In addition, a smooth muscle abnormality in the conduit arteries of these children cannot be excluded. It should be noted, however, that the dilation to GTN in the FH group was sufficiently great $(10 \pm 0.6 \%)$ so as not to preclude endothelium-dependent dilation.

Impaired flow-mediated dilation was found in three children in the control group. As the process of atherosclerosis begins in childhood and adolescence (48), even in normocholesterolemic subjects, these children may indeed already have abnormal arteries. Serial evaluation during long-term followup will be required to investigate this possibility.

In this study we investigated vascular physiology in the superficial femoral artery of children. In previous work we have defined the relationship between vessel size and FMD in systemic arteries in humans and found that the diameter of the SFA in children is within the optimal range for resolution and response (17). We have not studied the coronary arteries as these are not accessible to external ultrasound. Atherosclerosis, however, is a diffuse process, and clinical and autopsy data suggest that the disease often follows a parallel course in the coronary and proximal femoral arteries $(49,50)$. Other investigators have studied proximal segments of the femoral arteries to monitor the effects of lipid-lowering therapy on the regression of atherosclerotic lesions (51). It is likely that impaired endothelium-dependent dilation in the SFA of our children with $\mathrm{FH}$ reflects abnormalities of other sites in the arterial tree such as the carotid and coronary arteries.

In established disease, cholesterol-lowering therapy can attenuate progression of human coronary and femoral atherosclerosis and can lead to regression of existing plaques (51-53). In early disease, cholesterol-lowering therapy might be beneficial if it was associated with an improvement in endothelial function. In a primate model of early atherosclerosis, cholesterolinduced endothelial dysfunction has been reversed by reversion to a low-fat diet (10), and similar findings have recently been reported in adult men (35), using cholesterol-lowering drug therapy. In humans, the reduction in LDL cholesterol produced by a low-fat, low-cholesterol diet is small (54) and, despite the addition of lipid-lowering drugs in some of our patients, none had cholesterol or LDL levels as low as those shown to be beneficial in atherosclerosis regression studies in adults. There is legitimate concern about the administration of 
lifelong therapy with cholesterol-lowering agents (55) and many pediatricians therefore avoid their use (56). Our findings suggest that therapy may be especially important in children with $\mathrm{FH}$ who also have an elevated $\mathrm{Lp}$ (a) level, as this group may have a particularly high risk of atherosclerosis. Treatment is currently available to lower LDL cholesterol but not $\operatorname{Lp}(\mathrm{a})$ levels. Whether the early abnormalities in arterial physiology can be reversed with any intervention will require prospective investigation, which is facilitated by the availability of a noninvasive test for the study of endothelial and smooth muscle function.

\section{Acknowledgments}

The authors thank Dr. C. Bull for assistance with statistical analysis, and J. Robinson, O. Thomas, and S. McCarthy for technical assistance.

Dr. Sorensen is supported by the Danish Heart Foundation, Dr. Celermajer by the British Heart Foundation and Dr. Georgakopoulos by the European Society of Cardiology. This research was funded in part by a grant from Corda.

\section{References}

1. Muller, C. 1939. Angina pectoris in hereditary xanthomatosis. Arch. Intern. Med. 64:675-700.

2. Stary, H. C. 1989. Evolution and progression of atherosclerotic lesions in coronary arteries of children and young adults. Arteriosclerosis. 9(Suppl. I):1932.

3. Pathobiological Determinants of Atheroscelrosis in the Youth (PDAY) Research Group. 1990. Relationship of atherosclerosis in young men to serum lipoprotein cholesterol concentrations and smoking. JAMA (J. Am. Med. Assoc.). 264:3018-3024.

4. Kannel, W. B. 1978. Hypertension, blood lipids and cigarette smoking as co-risk factors for coronary heart disease. Ann. NY Acad. Sci. 304:128-139.

5. Klag, M. J., D. E. Ford, L. A. Mead, J. He, P. K. Whelton, K.-Y. Liang, and D. M. Levine. 1993. Serum cholesterol in young men and subsequent cardiovascular disease. N. Engl. J. Med. 328:313-318.

6. Armstrong, V. M., P. Cremer, P. Eberle, A. Manke, F. Schulze, H. Wieland, H. Kreauzer, and D. Seidel. 1986. The association between serum Lp(a) concentrations and angiography assessed coronary atherosclerosis: dependence on serum LDL levels. Atherosclerosis. 62:249-257.

7. Ross, R. 1986. The pathogenesis of atherosclerosis. N. Engl. J. Med. 314:488-500.

8. Henderson, A. H. 1991. Endothelium in control. Br. Heart J. 65:116-125.

9. McLenachan, J. M., J. A. Vita, R. D. Fish, C. B. Treasure, D. A. Cox, P. Ganz, and A. P. Selwyn. 1990. Early evidence of endothelial dysfunction at coronary branchpoints. Circulation. 82:1169-1173.

10. Harrison, D. G., M. L. Armstrong, P. C. Freiman, and D. D. Heistad. 1987. Restoration of endothelium-dependent relaxation by dietary treatment of atherosclerosis. J. Clin. Invest. 80:1808-1811.

11. Fish, R. D., E. G. Nabel, A. P. Selwyn, P. L. Ludmer, G. H. Mudge, J. M. Kirshenbaum, F. J. Schoen, R. W. Alexander, and P. Ganz. 1988. Responses of coronary arteries of cardiac transplant patients to acetylcholine. J. Clin. Invest. $81: 21-31$.

12. Galle, J., E. Bassenge, and R. Busse. 1990. Oxidized low density lipoproteins potentiate vasoconstrictions to various agonists by direct interaction with vascular smooth muscle. Circ. Res. 66:1287-1293.

13. Drexler, H., A. M. Zeiher, K. Meinzer, and H. Just. 1991. Correction of endothelial dysfunction in coronary microcirculation of hypercholesterolemic patients by L-arginine. Lancet. 338:1546-1550.

14. Vita, J. A., C. B. Treasure, E. G. Nabel, J. M. McLenachan, R. D. Fish, A. C. Yeung, V. I. Vekstein, A. P. Selwyn, and P. Ganz. 1990. Coronary vasomotor response to acetylcholine relates to risk factors for coronary artery disease. Circulation. 81:491-497.

15. Scientific Steering Committee on Behalf of the Simon Broome Register Group. 1991. Risk of fatal coronary heart disease in familial hypercholesterolaemia. Br. Med. J. 303:893-896.

16. Friedwald, W. T., R. I. Levy, and D. S. Fredrickson. 1972. Estimation of the concentration of low density lipoprotein cholesterol in plasma without the use of the preparative ultracentrifuge. Clin. Chem. 18:499-502.

17. Celermajer, D. C., K. E. Sorensen, V. M. Gooch, D. J. Spiegelhalter, O. I. Miller, I. D. Sullivan, J. K. Lloyd, and J. E. Deanfield. 1992. Non-invasive detection of endothelial dysfunction in children and adults at risk of atherosclerosis. Lancet. 340:1111-1115.
18. Celermajer, D. S., K. E. Sorensen, M. Ryalls, O. Thomas, J. Robinson, J. V. Leonard, and J. E. Deanfield. 1993. Endothelial dysfunction occurs in the systemic arteries of children with homozygous homocystinuria but not in their heterozygous parents. J. Am. Coll. Cardiol. 22:854-858.

19. Salonen, R., and J. T. Salonen. 1991. Determinants of carotid intima-media thickness: a population based ultrasonography study in Eastern Finnish men. J. Intern. Med. 229:225-231.

20. Wendelhag, I., O. Wiklund, and J. Wikstrand. 1992. Arterial wall thickness in hypercholesterolemia. Arterioscler. Thromb. 12:70-77.

21. Kwiterovich, P. O. Diagnosis and management of familial dyslipoproteinemia in children and adolescents. 1990. Pediatr. Clin. North Am. 37:1489-1523.

22. Kwiterovich, P. O., R. I. Levy, and D. S. Fredrickson. 1973. Neonatal diagnosis of familial type II hyperlipoproteinemia. Lancet. i:118-122.

23. Pohl, U., J. Hultz, R. Busse, and E. Bassenge. 1986. Crucial role of endothelium in the vasodilator response to increased flow in-vivo. Hypertension. $8: 37-44$.

24. Rubanyi, G. M., C. Romero, and P. M. Vanhoutte. 1986. Flow-induced release of endothelium derived relaxing factor. Am. J. Physiol. 250:1115-1119.

25. Cooke, J. P., A. H. Singer, P. Tsao, P. Zera, R. A. Rowan, and M. E. Billingham. 1992. Antiatherogenic effects of L-arginine in the hypercholesterolemic rabbit. J. Clin. Invest. 90:1168-1172.

26. Wines, P. A., J. M. Schmitz, S. L. Pfister, F. J. Clubb, Jr., L. M. Buja, J. T. Willerson, and W. B. Campbell. 1989. Augmented vasoconstrictor responses to serotonin precede development of atherosclerosis in aorta of WHHL rabbits. Arteriosclerosis. 9:195-202.

27. Komori, K., H. Shimokawa, and P. M. Vanhoutte. 1989. Hypercholesterolemia impairs endothelium-dependent relaxations to aggregating platelets in porcine iliac arteries. J. Vasc. Surg. 10:318-325.

28. Cohen, R. A., K. M. Zitnay, C. C. Haudenschild, and L. D. Cunningham. 1988. Loss of selective endothelial cell vasoactive functions caused by hypercholesterolemia in pig coronary arteries. Circ. Res. 63:903-910.

29. Kugiyama, K., S. A. Kerns, J. D. Morrisett, R. Roberts, and P. D. Henry. 1990. Impairment of endothelium-dependent relaxation by lysolecithin in modified low-density lipoproteins. Nature (Lond.). 344:160-162.

30. Tomita, T., M. Ezaki, M. Miwa, K. Nakamura, and Y. Inoue. 1990. Rapid and reversible inhibition by low density lipoprotein of the endothelium-dependent relaxation to hemostatic substances in porcine coronary arteries. Circ. Res. 66:18-27.

31. Jacobs, M., F. Plane, and K. R. Bruckdorfer. 1990. Native and oxidized low-density lipoproteins have different inhibitory effects on endothelium-derived relaxing factor in the rabbit aorta. Br. J. Pharmacol. 100:21-26.

32. Simon, B. C., L. D. Cunningham, and R. A. Cohen. 1990. Oxidized low density lipoproteins cause contraction and inhibit endothelium-dependent relaxation in the pig coronary artery. J. Clin. Invest. 86:75-79.

33. Yamamoto, H., C. Bossaller, J. Cartwright, 'Jr., and P. D. Henry. 1988. Videomicroscopic demonstration of defective cholinergic arteriolar vasodilation in atherosclerotic rabbit. J. Clin. Invest. 81:1752-1758.

34. Zeiher, A. M., H. Drexler, H. Wollschlager, and H. Just. 1991. Modulation of coronary vasomotor tone in humans. Circulation. 83:391-401.

35. Leung, W.-H., C.-P. Lau, and C.-K. Wong. 1993. Beneficial effect of cholesterol-lowering therapy on coronary endothelium-dependent relaxation in hypercholesterolaemic patients. Lancet. 341:1496-1500.

36. Scanu, A. M. 1992. Lipoprotein(a): a genetic risk factor for premature coronary heart disease. JAMA (J. Am. Med. Assoc.). 267:3326-3329.

37. Sandholzer, Ch., E. Boerwinkle, N. Saha, M. C. Tong, and G. Utermann 1992. Apolipoprotein (a) phenotypes, $\mathrm{Lp}$ (a) concentration and plasma lipid levels in relation to coronary heart disease in a Chinese population. Evidence for the role of the apo(a) gene in coronary heart disease. J. Clin. Invest. 89:1040-1046.

38. Genest, J., Jr., J. R. McNamara, J. M. Ordovas, J. L. Jenner, S. R. Silberman, K. M. Anderson, P. W. F. Wilson, D. N. Salem, and E. J. Schaefer. 1992. Lipoprotein cholesterol, apolipoprotein A-1 and B and lipoprotein (a) abnormalities in men with premature coronary artery disease. J. Am. Coll. Cardiol. 19:792802.

39. Seed, M., F. Hopplicher, D. Reaveley, S. McCarthy, G. Thompson, E. Boerwinkle, and G. Uterman. 1990. Relation of serum lipoprotein (a) concentration and apolipo-protein (a) phenotype to coronary heart disease in patients with familial hypercholesterolemia. N. Engl. J. Med. 322:1494-1499.

40. Wiklund, O., B. Angelin, S. Olufson, M. Erisson, G. Fager, L. Berglund, and G. Bonders. 1991. Apolipoprotein (a) and ischaemic heart disease in familia hypercholesterolemia. Lancet. ii:1360-1363.

41. Eaton, D. L., G. M. Fless, and W. J. Kohn. 1987. Partial amino-acid sequence of apolipoprotein (a) shows that it is homologous to plasminogen. Proc. Natl. Acad. Sci. USA. 84:3224-3228.

42. Laurent, S., P. Lacolley, P. Brunel, B. Laloux, B. Pannier, and M. Safar. 1990. Flow-dependent vasodilation of brachial artery in essential hypertension. Am. J. Physiol. 258:H1004-H1011.

43. Anderson, E. A., and A. L. Mark. 1989. Flow-mediated and reflex changes in large peripheral artery tone in humans. Circulation. 79:93-100.

44. Drexler, H., and A. M. Zeiher. 1991. Endothelial function in human coronary arteries in vivo. Hypertension (Dallas). 18:1190-1199. 
45. Liao, J. K., M. A. Bettmann, T. Sandor, J. I. Tucker, S. M. Coleman, and M. A. Creager. 1991. Differential impairment of vasodilator responsiveness of peripheral resistance and conduit vessels in humans with atherosclerosis. Circ. Res. 68:1027-1034.

46. Schnaar, R. L., and H. V. Sparks. 1972. Response of large and small coronary arteries to nitroglycerine, $\mathrm{NaNO}_{2}$, and adenosine. Am. J. Physiol. 223:223-228.

47. Mugge, A., J. H. Elwell, T. E. Peterson, T. G. Hofmeyer, D. D. Heistad. and D. G. Harrison. 1991. Chronic treatment with polyethylene-glycolated superoxide dismutase partially restores endothelium-dependent vascular relaxations in cholesterol-fed rabbits. Circ. Res. 69:1293-1300.

48. Enos, W. F., R. H. Holmes, and J. Beyer. 1953. Coronary disease among United States soldiers killed in action in Korea: preliminary report. $J A M A(J$. Am. Med. Assoc.). 153:1090-1092.

49. Olsson. A. G. 1991. Regression of femoral atherosclerosis. Circulation. 83:698-700.

50. Solberg, L. A., and J. P. Strong. 1983. Risk factors and atherosclerotic lesions. Arteriosclerosis. 3:187-198.

51. Blankenhorn, D. H., S. P. Azen. D. W. Crawford, S. A. Nessim, M. E.
Sanmarco, R. H. Selzer, A. M. Shircore, and E. C. Wickham. 1991. Effects of colestipol-niacin therapy on human femoral atherosclerosis. Circulation. 83:438447.

52. Duffield, R. G. T., B. Lewis, N. E. Miller, C. W. Jamieson, J. N. Brunt, and A. C. Colchester. 1986. Treatment of hyperlipidaemia retards progression of symptomatic femoral atherosclerosis. Lancet. ii:639-642.

53. Lichtlen, P. R., P. Nikutta, S. Jost, J. Deckers, B. Wiese, and W. Rafflenbeul. 1992. Anatomical progression of coronary artery disease in humans as seen by prospective, repeated, quantitated coronary angiography. Circulation 86:828-838.

54. Hunninghake, D. B., E. A. Stein, C. A. Dujovne, W. S. Harris, E. B. Feldman. V. T. Miller, J. A. Tobert. P. M. Laskarzewski, E. Quitter, J. Held, et al. 1993. The efficacy of intensive dietary therapy alone or combined with lovastatin in outpatients with hypercholesterolemia. N. Engl. J. Med. 328:1213-1219.

55. Newman, T. B., and W. S. Browner. 1992. Childhood cholesterol screening: contraindicated. JAMA (J. Am. Med. Assoc.) 267:100-101.

56. Kimm. S. Y. S., G. H. Payne, E. Lakatos, L. S. Webber, and J. Greenblatt. 1992. Primary care physicians and children's blood cholesterol. Prev. Med. 21:191-202. 